\title{
TREMOR ESSENCIAL
}

\section{CARACTERIZAÇÃO CLÍNICA DE UMA AMOSTRA DE 176 PACIENTES}

\author{
VANDERCI BORGES*, HENRIQUE BALLALAI FERRAZ** , LUIZ AUGUSTO FRANCO DE ANDRADE***
}

\begin{abstract}
RESUMO - Revisamos os registros de 176 pacientes com tremor essencial (TE) quanto à história familiar, características do tremor, distribuição anatômica, idade de início dos sintomas. Os pacientes foram divididos em dois grupos: familiar (F) e não familiar (NF). História familiar de tremor foi encontrada em $47,2 \%$ da amostra. $O$ tremor de ação e postura ocorreu combinadamente em $49,4 \%$, somente de postura em $26,7 \%$ e somente de ação em $9,1 \%$. $\mathrm{O}$ tremor de repouso foi registrado em $10,2 \%$, sempre associado a outras características. Não houve diferenças clínicas entre os grupos $\mathrm{Fe} N \mathrm{NF}$. As mãos foram acometidas em 94,9\% e a cabeça em 26,5\%. O acometimento isolado das mãos foi mais frequente no sexo masculino, enquanto o tremor de cabeça foi mais frequente no sexo feminino. A idade de início variou de 4 a 85 anos e se mostrou significantemente menor nos pacientes do sexo masculino com história familiar ( 36,5 anos) e também nos pacientes do sexo masculino com tremor de ação e postura combinados. A idade de início não foi diferente quando se compararam grupos de pacientes com a mãe ou o pai acometidos. Embora existam diferenças nesta amostra, elas não são suficientes para considerar o TE familiar e o não familiar como entidades distintas.
\end{abstract}

PALAVRAS-CHAVE: tremor essencial, características clínicas, hereditariedade.

Essential tremor: clinical evaluation of 176 patients.

SUMMARY - We reviewed the clinical records of 176 patients with essential tremor (ET) according to sex, age of onset, family history, tremor characteristics and body distribution. The patients were divided into two groups: familial (F) and non familial (NF). A positive family history for tremor was observed in $47,2 \%$ of the patients. Action and postural tremor were the most frequent characteristic $(49,4 \%)$, postural alone in $26,7 \%$ and action alone in $9,1 \%$. Rest tremor was recorded in $10,2 \%$, always associated with other characteristics. There were no clinical differences between the F and NF groups. Hands were involved in $94,9 \%$ and the head in $26,5 \%$. Isolated tremor of the hands was the most frequent form of presentation with some greater frequency in males. Sixty per cent of the patients who presented head tremor either isolated or not, were females. The presence or absence of family history did not show difference in this proportion. The age of onset ranged between 4 and 85 years. In the $F$ group a lower mean of onset ( 36,5 years) was seen in males with statistical significance. In male patients with combined action and postural tremor a lower age of onset was found. The fact of a patient having either parent affected by ET produced no difference in the age of onset. Familial or non familial ET bearing some differences, may not be considered as distinct entities.

KEY WORDS: essential tremor, clinical characteristics, genetics.

Tremor é uma oscilação rítmica de uma determinada parte do corpo decorrente de contrações de músculos antagonistas e podem ser síncronas ou alternantes ${ }^{7,12}$. Durante muitos séculos, vários autores tentaram definir os diversos tipos de tremores, mas foi James Parkinson em $1817^{21}$ no seu

Setor de Investigação de Moléstias Extrapiramidais do Departamento de Neurologia e Neurocirurgia da Escola Paulista de Medicina (EPM): *Pós-Graduanda; **Médico Assistente-Doutor; ***Professor Adjunto. Este estudo é uma súmula da Dissertação de Mestrado apresentada ao Curso de Pós Graduação em Neurologia da Escola Paulista de Medicina em 1993. Aceite: 21-outubro-1993. 
artigo 'An Essay on the Shaking Palsy', quem conseguiu fazer uma separação bem definida do tremor de repouso com os outros tipos de tremores. Dana ${ }^{6}$, em 1887, descreveu as características de três famílias com tremor hereditário e que foi denominado por ele como "neurasthenic tremor". Minor $^{5}$ (1922) fez as descrições com maiores detalhes de pacientes e seus ascendentes com tremor essencial e após este estudo denominou-se este tipo de tremor como do tipo "Minor". O tremor essencial (TE) é conceituado como distúrbio monossintomático caracterizado por tremor que aparece em ação e postura, podendo acometer qualquer parte do corpo, embora as mãos sejam o local mais frequentemente acometido. OTE tem início lento e insidioso em qualquer idade sendo mais frequente após os 40 anos. Homens e mulheres são acometidos igualmente ${ }^{5,23,19}$. A frequência do tremor varia de 5 a 7 ciclos por segundo, mas podem-se encontrar frequências maiores. Além dessas características, inclui-se história familiar de tremor em torno de $50 \%$ dos casos sendo a herança do tipo autossômica dominante com penetrância variável. Melhora com ingestão de álcool e drogas betabloqueadoras é habitualmente observada.

Por se tratar de um distúrbio comum e pelas características clínicas e epidemiológicas terem sido pouco estudadas em nossa população ${ }^{22}$, decidimos realizar este estudo.

\section{MATERIAL E MÉTODOS}

Foram revisados os prontuários de 176 pacientes. Destes, 126 eram procedentes do ambulatório do Setor de Investigação em Moléstias Extrapiramidais da Disciplina de Neurologia da Escola Paulista de Medicina no período de janeiro-1982 a dezembro-1989 e os 50 restantes eram provenientes da clínica particular de um de nós, no período de janeiro-1978 a maio-1989. Os pacientes foram distribuídos em dois grupos; familiar e não familiar. Foram

Tabela 1. Distribuição de 176 pacientes com tremor essencial nos grupos familiar e não familiar quanto ao sexo e idade de início.

\begin{tabular}{|c|c|c|c|c|c|c|}
\hline & \multicolumn{3}{|c|}{$\begin{array}{c}\text { Grupo } \\
\text { Familiar }\end{array}$} & \multicolumn{3}{|c|}{$\begin{array}{c}\text { Grupo } \\
\text { Não Familiar } \\
\end{array}$} \\
\hline & $\mathrm{n}$ & $\%$ & ini & $\mathrm{n}$ & $\%$ & ini \\
\hline Masculino & 36 & {$[41,4]$} & $36,5^{*}$ & 51 & {$[58,6]$} & 56,0 \\
\hline Feminino & 47 & {$[52,8]$} & 52,0 & 42 & {$[47,2]$} & 55,6 \\
\hline Total & 83 & {$[47,2]$} & & 93 & {$[52,8]$} & \\
\hline
\end{tabular}

n, número de pacientes; ini, idade de início (em anos); ${ }^{*} \mathrm{p}<0,05$. considerados integrantes do grupo familiar os pacientes que tinham pelo menos um parente acometido.

Os dados destes pacientes preeenchiam os critérios clínicos de diagnóstico de TE segundo os critérios de Marsden et al. ${ }^{17}$ que foram modificados. Estes critérios são: ausência de tremor de repouso; tremor que aparece durante a manutenção de postura como a extensão dos membros superiores; o tremor não piora excessivamente com o movimento apesar de persistir durante ele; ausência de sinais parkinsonianos ou cerebelares associados. As modificações acrescentadas foram a associação de tremor de repouso com ação e/ou postura.

Os critérios de exclusão foram: pacientes cujos dados de prontuário eram incompletos, ausência de tremor

Tabela 2. Características do tremor de acordo com o sexo e idade de início.

\begin{tabular}{|c|c|c|c|c|c|c|}
\hline \multirow{2}{*}{ Característica } & \multicolumn{3}{|c|}{ Masculino } & \multicolumn{3}{|c|}{ Feminino } \\
\hline & $\mathrm{n}$ & $\%$ & ini & $\mathbf{n}$ & $\%$ & ini \\
\hline Ação & 10 & {$[11,2]$} & 57,9 & 6 & {$[6,9]$} & 40,2 \\
\hline Postural & 17 & {$[19,1]$} & 42,7 & 30 & {$[34,5]$} & 52,5 \\
\hline Ação e Postural & 51 & {$[57,3]$} & 46,5 & 36 & {$[41,4]$} & 55,2 \\
\hline Outros & 11 & {$[12,4]$} & 52,7 & 15 & {$[17,2]$} & 57,8 \\
\hline Total & 93 & {$[53,4]$} & & 83 & {$[47,6]$} & \\
\hline
\end{tabular}

$\mathrm{n}$, número de pacientes; ini, idade de início (em anos). Outros: $\mathrm{A}+\mathrm{R}, \mathrm{P}+\mathrm{R}, \mathrm{A}+\mathrm{R}+\mathrm{P}, \mathrm{A}+\mathrm{I}$. $\mathrm{A}$, ação; $\mathrm{P}$, postural;

$R$, repouso; I, intencional. 
Tabela 3. Distribuição dos pacientes quanto ao acometimento isolado das mãos e do segmento cefálico em relação aos outros segmentos.

\begin{tabular}{lccccccccc}
\hline & \multicolumn{2}{c}{ Masculino } & & \multicolumn{2}{c}{ Feminino } & & \multicolumn{2}{c}{ Total } \\
\cline { 2 - 3 } \cline { 8 - 10 } & $\mathrm{n}$ & $\%$ & & $\mathrm{n}$ & $\%$ & & $\mathrm{n}$ & $\%$ \\
\hline \hline Mãos somente & 69 & {$[59,0]^{*}$} & & 48 & {$[41,0]$} & & 117 & {$[100]$} \\
Cabeça & 13 & {$[27,7]$} & & 34 & {$[72,3]^{*}$} & & 47 & {$[100]$} \\
Outras & 5 & {$[41,7]$} & & 7 & {$[58,3]$} & & 12 & {$[100]$} \\
Total & 87 & {$[49,4]$} & & 89 & {$[50,6]$} & & 176 & {$[100]$} \\
\hline
\end{tabular}

n, número de pacientes; Outras, mãos associado a outro segmento corporal que não o segmento cefálico; ${ }^{*} \mathrm{p}<0,01$.

à primeira consulta, hipertireoidismo, uso de drogas indutoras de tremor, tremor intencional ou de repouso puros; presença de movimentos involuntários associados, pacientes que apresentavam tremor em um tipo particular de função (tremor tarefa-específica) e diagnósticos duvidosos.

Os dados analisados foram: sexo, história familiar, características do tremor tanto isoladas ou combinadas; segmentos corporais afetados; e se ocorriam isolados ou associados, idade de início. Foram anotados também o uso pregresso de drogas antiparkinsonianas e o uso e melhora com álcool.

\section{RESULTADOS}

Dos 176 pacientes, 87 eram do sexo masculino e 89 do sexo feminino. Os pacientes do grupo familiar eram 83 ( 36 homens, 47 mulheres) e do grupo não familiar 93 ( 51 homens, 42 mulheres) (Tabela 1). Não se observou diferença estatísticamente siginificante entre os sexos.

Na distribuição dos pacientes segundo as características do tremor em relação ao sexo observou-se que o tremor de ação e postura associados ocorreu em cerca de metade dos pacientes, a característica postural em $26,7 \%$ e ação em 9,1\%. Dos pacientes, $10 \%$ apresentavam tremor de repouso associado a alguma outra forma (Tabela 2). Ao se separar nos grupos familiar e não familiar os resultados foram semelhantes.

O acometimento das mãos ocorreu na maioria (167) dos pacientes $(94,8 \%)$ seguido da região cefálica em 47 $(26,7 \%)$. As demais localizações que foram os membros inferiores, mento, aparelho fonador, tronco e língua somaram $31(17,6 \%)$. As formas de acometimento mais frequentes foram as mãos de maneira isolada $(66,5 \%)$ seguida da associação mãos e cabeça $(14,7 \%)$. Foi possível observar que o tremor isolado das mãos era significantemente mais frequente no sexo masculino (Tabela 3) e o acometimento do segmento cefálico tanto isolado como associado predominava no sexo feminino (Tabela 3 ).

A idade de início variou de 4 a 85 anos com média de 45 anos. No sexo masculino a média da idade de início foi 47,8 anos e no feminino 53,9 anos. No grupo familiar (F) a idade média de início foi significantemente menor no sexo masculino do que no feminino (Tabela 1). No grupo não familiar observou-se uma concentração na $7^{7}$ década em ambos os sexos, enquanto no grupo não familiar (NF) ocorreu um pico de concentração na $7^{\mathbf{a}}$ década no sexo feminino e no masculino nas $2^{\mathrm{a}}$ e $6^{\mathrm{a}}$ décadas. Analisando a idade de início em relação às características do tremor, foi notado que no sexo masculino com tremor de ação e postural combinados, o início era significantemente mais precoce. A idade de início também foi significantemente menor nos homens do grupo familiar com tremor exclusivo das mãos.

A melhora com ingestão de álcool foi obtida em $12,5 \%$ dos pacientes. Observou-se que a herança materna ou paterna não foi determinante para a idade de início do tremor. Nenhum dos pacientes desta amostra que permaneceram em acompanhamento desenvolveu parkinsonismo.

\section{COMENTÁRIOS}

Neste estudo foram incluídos os dados dos pacientes cujos prontuários apresentavam informações corretas e se enquadravam no diagnóstico de TE de acordo com a classificação de Marsden et. $\mathrm{al}^{17}$ com algumas modificações efetuadas.

A ausência de diferença entre o número de pacientes nos dois sexos nos grupos $\mathrm{F}$ e NF foi semelhante ao encontrado em outros estudos ${ }^{5,10,19,23}$. Rautakorpi et al. ${ }^{24}$, por outro lado, encontraram 
uma maior frequência no sexo masculino e Hornabrook e Nagurney ${ }^{11}$, Haerer et al..$^{9}$ e Pereira et al. ${ }^{22}$ encontraram maior frequência no sexo feminino.

A característica mais frequente foi a combinação do tremor de ação e postura. Os tremores de ação ou de postura puros aparecem menos frequentemente. Biary e Koller ${ }^{2}$ descreveram a presença do tremor de ação acentuado com mínima ou nenhuma característica postural em $10,5 \%$ de sua população de TE. O tremor de repouso, associado a outras características, como foi por nós observado, já foi descrito em outras amostras de $T^{13,19}$ Este tipo de tremor não é exclusivo da doença de Parkinson, podendo ser visto no TE, tremor rubral, doenças heredodegenerativas. Por isso a sua presença não invalida o diagnóstico.

Em relação ao segmento corporal, o tremor das mãos apareceu na maioria dos pacientes, sendo mais frequente a forma isolada e predominando no sexo masculino, seguido da associação de mãos e cabeça. A maior frequência do tremor cefálico em mulheres, observada nesta amostra, ocorreu tanto isoladamente como em combinação com outros segmentos. A observação de que o tremor cefálico é mais frequente em mulheres não havia sido descrita antes. Estudos que levem a um melhor conhecimento da fisiopatologia do TE poderão dar uma explicação para este achado.

Ao contrário do que foi por nós encontrado, Hornabrook e Nagurney"1 descreveram que o tremor se manifestou mais frequentemente nas mãos e cabeça em associação, mas não fazem menção de existir diferença entre os sexos. As mãos foram os segmentos corporais mais afetados em outras amostras ${ }^{10,16,22}$. Larsson e Sjögren observaram presença do tremor cefálico em $23,3 \%$ sem diferença entre os sexos ${ }^{14}$. Estudando-se o grupo familiar e não familiar, foi observado que a proporção de acometimento das mãos era igual em ambos os sexos e o acometimento do segmento cefálico se mostrou mais frequente nas mulheres. Também foi notado no grupo familiar que o sexo feminino apresentava maior quantidade de associações de segmentos corporais com tremor em contraposição ao grupo não familiar em que a distribuição foi semelhante. História familiar de tremor foi observada em cerca de metade da amostra e é semelhante ao observado na literatura, que tem mostrado proporções variando de 39 a $62,5 \%^{16,19,23}$. É possível que a proporção por nós observada seja inferior à realidade, já que nós não examinamos os familiares dos pacientes, assim como muitos que negaram história familiar de tremor, na verdade podem desconhecer sua existência em alguns de seus parentes. Lou e Jankovic ${ }^{16}$ encontraram $62,5 \%$ de história familiar numa amostra de 350 pacientes e provavelmente isto ocorreu porque tiveram a oportunidade de examinar os parentes dos pacientes. Além disto, como a transmissão genética é autossômica dominante, com penetrância e expressividade variáveis, é possível que os parentes não tenham desenvolvido tremor em amplitude suficiente para atrai-los à atenção médica, o que também deve ter contribuído para menor frequência de história familiar.

A tendência dos pacientes do sexo masculino apresentarem o tremor localizado nas mãos, ao contrário dos do sexo feminino em que além de se manifestar nas mãos tende acometer outras partes do corpo pode ser devida a possível fator de proteção para a expressividade do gene nos homens.

A média de idade de início em nossos pacientes variou de 4 a 85 anos. Em outros estudos a média da idade de início variou de 35 a 50 anos $^{4,14,15,19}$ Destes estudos, só no de Larsson e Sjögren ${ }^{14}$ pode-se notar que a diferença na média da idade de início foi um pouco maior para as mulheres, embora não significante. $\mathrm{O}$ TE raramente é diagnosticado antes da adolescência. Porém, nesta amostra a informação do início antes dos 15 anos de idade foi obtida em 9 pacientes do sexo masculino do grupo familiar. Destes 9 pacientes 4 tiveram início antes dos 10 anos. Para saber se eram estes pacientes que provocaram o achado significante da diferença da média de idade no sexo masculino, foram retiradas estas idades do cálculo e ainda assim, obteve-se valor estatísticamente significante.

A idade de início em relação às características do tremor neste estudo só mostrou diferença significante nos pacientes do sexo masculino de ambos os grupos que demonstravam tremor de ação e postura associados. Martinelli et al ${ }^{19}$ não notaram diferença na idade de início dos pacientes que apresentavam características típicas de TE e tipos incomuns. Ao se considerar as formas de 
acometimento, nosso estudo mostrou que a média de idade de início foi mais precoce nos pacientes do sexo masculino do grupo familiar que apresentavam tremor isolado das mãos. Já foi relatado início do tremor mais precoce em tremor cefálico ${ }^{14}$, e em tremor das mãos ${ }^{16}$.

Sabe-se que a ingestão de álcool melhora o tremor por mecanismos desconhecidos ${ }^{1,8,12}$. A pequena frequência encontrada de melhora com a ingestão de álcool em nossa amostra, deveu-se ao fato dos pacientes, em sua maioria, negarem o uso e provavelmente por receio de serem denominados alcoólatras.

O fato de que o tipo de herança materna ou paterna não ser fator importante para o início do tremor já havia sido descrito por Larsson e Sjögren ${ }^{14}$, os quais determinaram a ausência do fenômeno de antecipação.

A não existência de desenvolvimento de parkinsonismo nesta amostra sugere não haver relação entre as duas entidades neste estudo. Este é assunto ainda controverso, pois alguns autores defendem a associação entre as duas entidades ${ }^{16,25}$ e outros não encontraram tal relação $0^{3,7,10,14,18}$

As diferenças existentes entre o tremor essencial familiar e tremor essencial não familiar nesta amostra não são suficientes para considerá-las como duas entidades clínicas distintas.

Agradecimentos: Os autores agradecem o auxílio do CNPq.

\section{REFERÊNCIAS}

1. Ashenhurst, EM. The nature of essential tremor. Can Med Assoc J 1973, 109: 876-878.

2. Biary N, Koller WC. Kinetic predominant essential tremor: succesful treatment with clonazepam. Neurology 1987, 37: 471-474.

3. Cleeves L, Findley LJ, Koller W. Lack of association between essential tremor and Parkinson's disease. Ann Neurol 1988, 24: 23-26.

4. Critchley E. Clinical manifestations of essential tremor. J Neurol Neurosurg Psychiatry 1972, 35: 365-372.

5. Critchley M. Observations on essential (heredofamilial) tremor. Brain 1949, 72: 113-139.

6. Dana CL. Hereditary tremor: a hitherto undescribed form of motor neurosis. Am J Med Sci 1887, 94: 386-393.

7. Findley LJ. Tremors: Differential diagnosis and pharmacology. In Jankovic, J, Tolosa E, (eds.) Parkinson's disease and movement disorders. Baltimore: Urban \& Schwarzenberg 1988 p 243-261.

8. Growdon JH, Shahani BT, Young RR. The effect of alcohol on essential tremor. Neurology 1975, 25: 259-262.

9. Haerer AF, Anderson DW, Schoenberg BS. Prevalence of essential tremor: results from the Copiah Country study. Arch Neurol 1982, 39: 750-751.

10. Herskovits E, Figueroa E, Mangone C. Hereditary essential tremor in Buenos Aires (Argentina), Arq Neuropsiquiatr 1988, 46: 238-247.

11. Hornabrook RW, Nagurney JT. Essential tremor in Papua New Guinea. Brain 1976, 99: 659-672.

12. Koller WC. Diagnosis and treatment of tremor. Neurol Clin 1984, 2: 499-514.

13. Koller WC, Rubino FA. Combined resting postural tremors. Arch Neurol 1985, 42: 683-684.

14. Larsson T, Sjögren T. Essential tremor: a clinical and genetic population study. Acta Psychiatr Neurol Scand 1960, 36 (Suppl 144): 1-176.

15. Longe AC. Essential tremor in Nigerians: a prospective study of 35 cases. East Afr Med J 1985, 62: 672-676.

16. Lou JS, Jankovic J. Essential tremor: clinical correlates in 350 patients. Neurology 1991, 41: 234-238.

17. Marsden CD, Obeso JA, Rothwell JC. Benign essential tremor is not a single entity. In: Yahr MD (ed.) Current concepts in Pakinson's disease. Amsterdam: Excerpta Medica, 1983, p 31-46.

18. Martilla RJ, Rautakorpi I, Rinne UK. The relation of essential tremor to Parkinson's disease. J Neurol Neurosurg Psychiatry 1984, 47: 734-735.

19. Martinelli P, Gabellini AS, Gulli MR, Lugaresi E. Different clinical features of essential tremor: a 200 patient sudy. Acta Neurol Scand1987, 75: 106-111.

20. Minor L. Uber hereditaren Tremor. Berl Ges f Psychiatrie u Nervenkrankh 1922, 13(III): 514-516.

21. Parkinson J. An essay on the shaking palsy. London: Sherwood, Neely \& Jones, 1817. Ed. fac-simile in Med Class 1983, 3: 964-997.

22. Pereira JS, Pimentel MLV, Nicaretta DH. Tremor essencial: observaçōes clínicas. Arq Neuropsiquiatr 1993,51:337-340.

23. Rajput AH, Offord KP, Beard CM, Kurland LT. Essential tremor in Rochester, Minnesota: a 45-year study. J Neurol Neurosurg Psychiatry 1984, 47: 466-470.

24. Rautakorpi I, Takala J, Marttila RJ, Sievers K, Rinne UK. Essential tremor in a Finnish population. Acta Neurol Scand 1982, 66: 58-67.

25. Roy M, Boyer L, Barbeau A. A prospective study of 50 cases of familial Parkinson's disease. Can J Neurol Sci 1983, 10: 37-42. 